\title{
Searches for Chargino-Neutralino Production at the Tevatron
}

\author{
Jared Yamaoka*t \\ Duke University \\ Department of Physics \\ Physics Bldg., Science Dr. \\ Box 90305 \\ Durham, NC 27708 \\ USA \\ E-mail: yamaoka@cern.ch
}

\begin{abstract}
The production of chargino-neutralino $\left(\chi_{1}^{ \pm} \chi_{2}^{0}\right)$ pairs and their subsequent leptonic decays is one of the most promising supersymmetry (SUSY) signatures at the Tevatron proton-antiproton collider. We present here the most recent results on the search for the three-lepton and missing transverse energy SUSY signature using data collected with the CDF and DØ detectors. At CDF, charginoneutralino pairs are also searched for using events with a $\mathrm{Z}$ (decaying to $e^{+} e^{-}$), two or more jets from a $\mathrm{W}$ decay, and large missing transverse energy. The presented results, based on data corresponding to $1.0-3.2 \mathrm{fb}^{-1}$ of integrated luminosity, are interpreted in the minimal supergravity scenario.
\end{abstract}

European Physical Society Europhysics Conference on High Energy Physics, EPS-HEP 2009, July 16 - 222009

Krakow, Poland

\footnotetext{
*Speaker.

†n behalf of the CDF and DØ Collaborations.
} 


\section{Introduction}

Supersymmetry (SUSY) [1] is one of the most popular extensions of the standard model (SM). SUSY can solve the hierarchy problem which the SM suffers from and allows the unification of the gauge couplings. Each SM particle has a corresponding SUSY particle (sparticles) that contributes to the Higgs mass squared with opposite sign relative to the contributions of SM particles. This protects the weak mass scale, $M_{W}$, from divergences. SUSY is a broken symmetry since the sparticles do not have the same mass as their SM partners. If $R_{p}$ parity is conserved, the lightest SUSY particle (LSP) is stable and provides a candidate for cosmological dark matter. We use as a reference the minimal super gravity (mSUGRA) model of SUSY breaking [2]. The mSUGRA scenario is described by five independent parameters: the unified scalar and gaugino masses $m_{0}$ and $m_{1 / 2}$, the ratio of the vacuum expectation values of the two Higgs doublets, $\tan \beta$, the unified trilinear coupling $A_{0}$, and the sign of the Higgs mass parameter $\mu$. These parameters determine all the properties of the model, e.g. masses and cross sections.

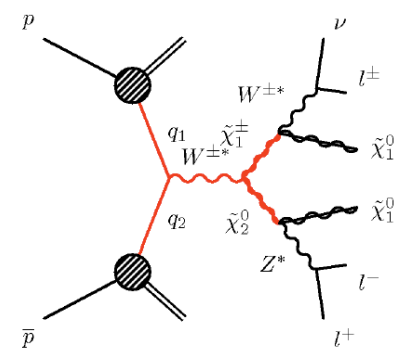

(a) Trilepton Channel

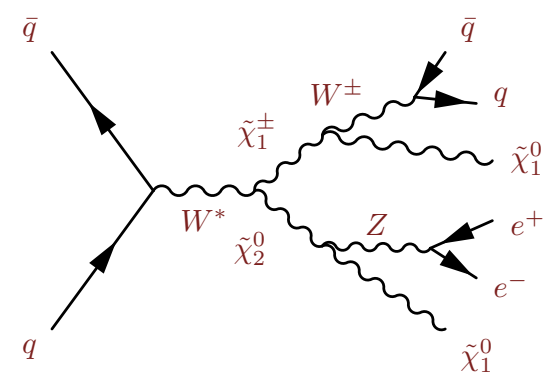

(b) Lepton, Jets, and $E_{T}$ Channel

Figure 1: Feynman diagrams for the decay channels discussed in this paper.

\section{Chargino-neutralino in trilepton final states}

The 'classical' signature for SUSY is the trilepton channel, Figure 1(a). The advantage of this channel is that the expected contributions from SM processes are very low for final states with three isolated leptons and large missing transverse energy $\left(E_{T}\right)$. The disadvantage is the SUSY processes leading to a trilepton signature has a low cross section times branching ratio. Furthermore, the leptons have relatively low transverse momentum $\left(p_{T}\right)$ so reconstructing them is more difficult.

\subsection{Analysis strategy at the CDF experiment}

In the CDF analysis [3,4], we select events and separate them, based on lepton type, into exclusive samples based on expected purity. This channel independence allows easy statistical combination of the results. For each event, we select tracks, ('tight' and 'loose') muons $(\mu)$, and electrons of some quality. Each of these objects, except the tracks, have tight and loose categories. We then assign each event to an exclusive channel composed of combinations of these objects. While all the channels are kept exclusive, this effectively gives two larger categories of events; those with three identified leptons (trileptons) and those with two leptons and a track (dilepton+track). We 


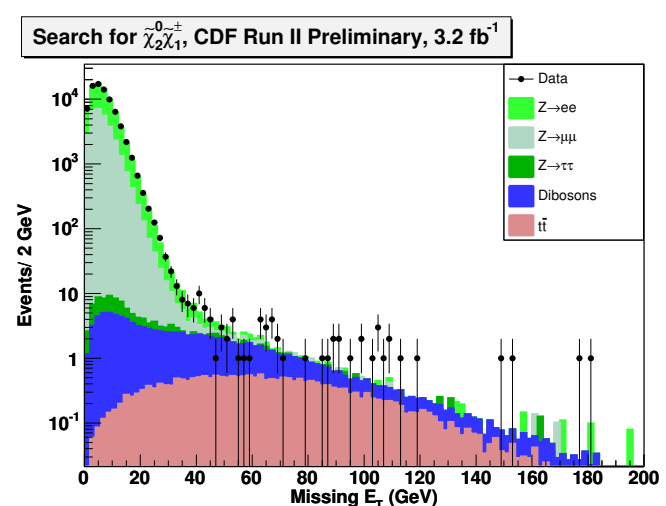

(a) $E_{T}$ dilepton control region for the $\mathrm{CDF}$ trilepton analysis.

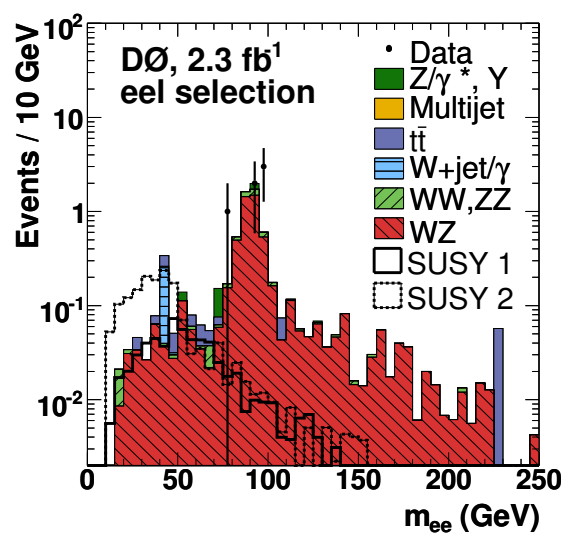

(b) Dielectron invariant mass eel channel. Data compared to SM prediction for the $\mathrm{D} \varnothing$ trilepton analysis.

Figure 2: Data comparisons for the $\mathrm{CDF}$ and $\mathrm{D} \emptyset$ trilepton analysis.

create two types of control samples to test our background estimations against data. The first type is a dilepton selection, which consists of the first two objects of the trilepton selection, Figure 2(a). The second type adds a third lepton. These trilepton events are chosen in a kinematic region where we do not expect any signal. Good agreement is found between data and SM predictions in all the control regions. We then apply SUSY specific cuts optimized for $m_{0}=60 \mathrm{GeV}, m_{1 / 2}=190 \mathrm{GeV}$, $\tan \beta=3, A_{0}=0$ and $\mu>0$, and look at signal region data to compare against background. This analysis is performed with data collected until 1 July, 2008, totaling $3.2 \mathrm{fb}^{-1}$.

\subsection{Analysis strategy at the $D \emptyset$ experiment}

In the DØ analysis [5], we define four different channels distinguished by the lepton content of the final state. For the dielectron plus lepton channel (eel) we require two identified electrons and a track. In the dimuon plus lepton channel $(\mu \mu l)$, we require one tight and one loose muon and a track. The selection in the electron, muon plus lepton channel $(e \mu l)$ requires one electron and one tight muon and a track. Finally, we select events with a tight muon, a hadronically decaying tau, and either a track $(\mu \tau l)$ or another hadronically decaying tau $(\mu \tau \tau)$. For each of the $e e l, \mu \mu l$ and $e \mu l$ channels, one "low- $\mathrm{p}_{T}$ " and one "high- $\mathrm{p}_{T}$ " selection is designed to exploit the different kinematic properties for different mass ranges. Primarily these selections tune the $p_{T}$ cuts of the leading leptons to maximize the expected sensitivity. The $\mu \tau l$ and $\mu \tau \tau$ channels use only one set of cuts similar to the low- $\mathrm{p}_{T}$ selection. The analysis is optimized using mSUGRA as a reference model. Two sets of mSUGRA parameters $\left(m_{0}=150 \mathrm{GeV}\right.$ and $m_{1 / 2}=250$ (170) GeV, with $\tan \beta=3$, $A_{0}=0$ and $\left.\mu>0\right)$ are used as a reference for a high- $\mathrm{p}_{T}\left(\right.$ low- $\mathrm{p}_{T}$ ) signal, labeled SUSY1 (SUSY2), Figure 2(b). This analysis is performed with data collected until June, 2007, totaling $2.3 \mathrm{fb}^{-1}$. The analysis using hadronic $\tau$ decays is based on $1.0 \mathrm{fb}^{-1}$.

\subsection{Results for the trilepton analysis}

Neither CDF nor DØ analyses see an excess of events relative to the SM predictions, Table 1. 


\begin{tabular}{|c|c|c|c|}
\hline & Channel & SM Expectation & data \\
\hline \hline CDF & trilepton & $1.5 \pm 0.2$ & 1 \\
& dilepton+track & $9.4 \pm 1.4$ & 6 \\
\hline DØ & Low $\mathrm{p}_{T}$ & $5.4 \pm 0.6$ & 9 \\
& High $\mathrm{p}_{T}$ & $3.3 \pm 0.4$ & 4 \\
\hline
\end{tabular}

Table 1: Standard Model expectation and data for the CDF and D $\varnothing$ trilepton analysis.

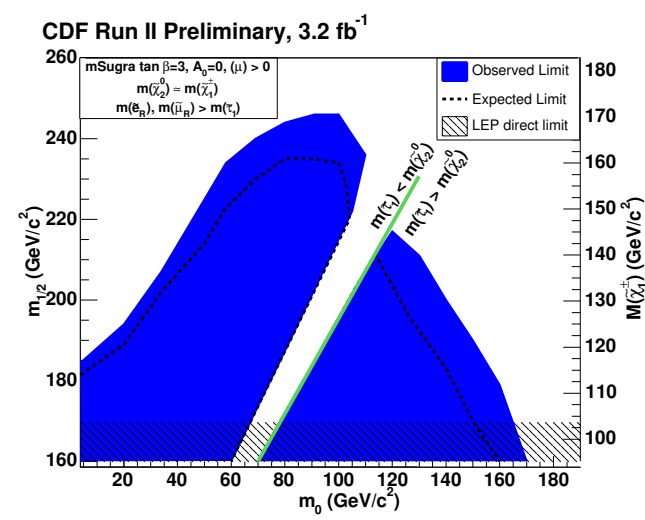

(a) CDF Exclusions in the $m_{1 / 2}-m_{0}$ plane.

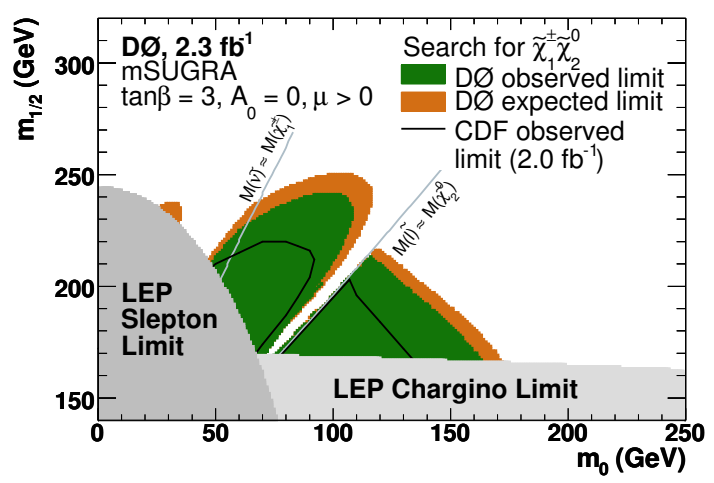

(b) DØ Exclusions in the $m_{1 / 2}-m_{0}$ plane.

Figure 3: Limits in the mSUGRA model from the trilepton analysis. $\left(\tan \beta=3, \mathrm{~A}_{0}=0\right.$, and $\left.\mu>0\right)$

However, we can turn these null results into exclusions on the mSUGRA model. Assuming a parameter space $\left(\mathrm{m}_{0}=60 \mathrm{GeV}, \tan \beta=3, \mathrm{~A}_{0}=0\right.$, and $\left.\mu>0\right)$ we exclude the chargino $\left(\chi_{1}^{ \pm}\right)$of a mass less than $155 \mathrm{GeV} / \mathrm{c}^{2}$ at $\mathrm{D} \emptyset$ and less than $164 \mathrm{GeV} / \mathrm{c}^{2}$ at $\mathrm{CDF}$. The results are interpreted in the mSUGRA scenario, and translated into limits on $m_{0}$ vs $m_{1 / 2}$, Figure 3.

\section{Chargino-neutralino in leptons, jets, and $E_{T}$ final states}

The aim of this analysis [6] is very similar to the trilepton analysis but designed to explore a different mSUGRA parameter space. The trilepton analysis includes decays through virtual $\mathrm{Z}^{0}$ and $\mathrm{W}^{ \pm}$, and thus can cover a lower mass region. The parameter space in the trilepton analysis also allows gauginos to decay through sleptons resulting in trilepton final state almost $100 \%$ of the time. Here we explore a higher mass range where the $\chi_{1}^{ \pm} \chi_{2}^{0}$ can decay via real $Z^{0}$ and $W^{ \pm}$ bosons, Figure 1(b). By requiring $\mathrm{Z}$ decaying to ee and $\mathrm{W}$ decaying to two jets, we exploit these resonances to reduce our backgrounds. We also require large missing energy coming from the LSP. The analysis optimizes the $E_{T}$ selection to obtain the best the expected limit. Figure 4(a) shows the data and SM background prediction. A test signal $\left(m_{0}=1000 \mathrm{GeV}\right.$ and $m_{1 / 2}=275 \mathrm{GeV}$, with $\tan \beta=10, A_{0}=0$ and $\left.\mu>0\right)$ is overlaid. This analysis is performed with data collected until February, 2008, totaling $2.68 \mathrm{fb}^{-1}$. Like the trilepton analysis there is no excess of events, Table 3 so we try to set a limit on the $\chi_{1}^{ \pm}$mass. (In this parameter space $m_{\chi_{1}^{ \pm}} \approx m_{\chi_{2}^{0}}$ ). Figure 4(b) shows we are not able to exclude any masses in the mSUGRA model. 


\begin{tabular}{|c|c|c|}
\hline Cut & SM Expectation & data \\
\hline \hline$E_{T}>40 \mathrm{GeV}$ & $6.4 \pm 0.9$ & 7 \\
$E_{T}>50 \mathrm{GeV}$ & $3.8 \pm 0.6$ & 2 \\
$E_{T}>60 \mathrm{GeV}$ & $2.0 \pm 0.3$ & 1 \\
\hline
\end{tabular}

Table 2: Standard Model expectation and data for the CDF Leptons $+\mathrm{Jets}+E_{T}^{\prime}$ analysis.

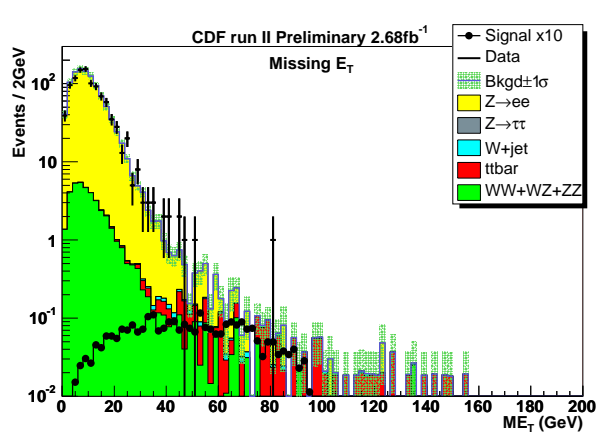

(a) Data compared to background for the CDF Lepton, Jets, and $E_{T}$ analysis.

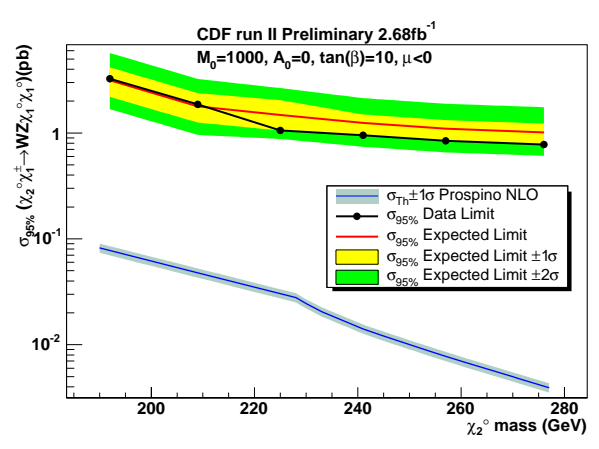

(b) CDF limits on mSUGRA

Figure 4: Limits from the CDF Lepton, Jets, and $E_{T}^{\prime}$ analysis.

\section{Conclusions}

The Tevatron is collecting high quality data at an unprecedented rate and both the CDF and $\mathrm{D} \emptyset$ experiments are doing their best to probe new models. Within the mSUGRA model we are able to exclude large areas of the parameter space using trilepton searches.

\section{References}

[1] S. P. Martin, A Supersymmetry Primer, [hep-ph/9709356].

[2] H. Baer, C. h. Chen, F. Paige and X. Tata, Phys. Rev. D 52, 2746 (1995) [hep-ph/9503271].

[3] T. Aaltonen et al. [CDF Collaboration], Phys. Rev. Lett. 101, 251801 (2008) [hep-ex/0808.2446].

[4] [CDF Collaboration], CDF SUSY Trilepton Search with $3.2 \mathrm{fb}^{-1}$, (2009) [http://www-cdf.fnal.gov/physics/exotic/r2a/20090521.trilepton_3fb/Welcome.html].

[5] V. M. Abazov et al. [D0 Collaboration], Search for associated production of charginos and neutralinos in the trilepton final state using $2.3 \mathrm{fb}-1$ of data, [hep-ex/0901.0646].

[6] [CDF Collaboration], CDF Gaugino Search using Z, W, and Missing $E_{T}$, (2009) [http://www-cdf.fnal.gov/physics/exotic/r2a/20090423.gaugino_wzmet/]. 